\title{
Informatic System for Monitoring Public Transportation in Urban Area
}

\author{
Cristina Ciurte $^{1}$, Adrian Florea ${ }^{1}$ \\ ${ }^{1}$ Computer Science and Electrical Engineering Department \\ "Lucian Blaga" University of Sibiu, Emil Cioran Street No. 4, Sibiu, Romania \\ \{ciurte.cristinaa@gmail.com\} \{adrian.florea@ulbsibiu.ro\}
}

\begin{abstract}
The number of cars is increasing and, in addition to this growth, people's needs for urban mobility are increasing. Changing travel habits, demand for services to increase comfort, speed and predictability, as well as evolving customer expectations for individualization and sustainability will require expansion of the portfolio of mobility services. This is why an urgent solution for this problem is mandatory to help citizens to travel safe and in time. This paper shows how an application can improve the way a person uses the public transport and helps to save time. The solution is to have an informatic system which monitors the public transport activity. This application, developed in Android Studio, informs users about the real-time position of the bus. In this way, the user will know how it is best to manage their time, whether they are waiting for the bus station, whether the bus is approaching the station or whether the time spent waiting for the bus in the bus station is too long and will choose other alternatives, such as walking, taking a taxi or another car.
\end{abstract}

Keywords: Smart Transportation, Public Transport, Real-life data, Informatic System

Received: 2 June 2019, Revised 9 September 2019, Accepted 20 September 2019

DOI: $10.6025 /$ jet/2019/10/2/38-45

(C) 2019 DLINE. All Rights Reserved

\section{Introduction}

Smart mobility is one of the challenges of modern society. The world's population is increasingly eager to live in the city. $53 \%$ of the population currently lives in urban areas and by 2050 this number is expected to reach $67 \%$. If all people living in urban areas travel with their private cars on a daily basis, the number will be extremely high and the capacity of the infrastructure will be exceeded, thus leading to congestion, time loss and air pollution from the gases emitted from cars [1].

The aim of the project is to design an application to help citizens who want to use public transport, resulting in traffic flow and also less polluted air. In order to decrease the cars' number, most people need to travel with public transport. However, in order for this to be comfortable for passengers, they must be informed every moment of the bus's location.

One way can be represented by creating an application to monitor public transport that follows the buses and provides users

\begin{tabular}{llllll}
\hline 38 & Journal of E - Technology Volume 10 & Number 2 & May 2019 \\
\hline
\end{tabular}


with their real-time location. In this way, both young and elderly people will be able to manage their time in a much more efficient way, without having to run after the bus or wait a long time at the station after them.

The main purpose of the application is to facilitate travel and thus attract more people to use public transport, thereby helping to reduce traffic and reduce emissions.

A method of reducing the number of vehicles present on a daily basis in traffic is to offer passengers other transport choices. One of them may be the public transport. An application to inform them at any time of the location of the desired buses is a solution for convincing more people to use this type of transport. In this way, the number of cars present, especially in peak hours, can decrease. Since travelers often face delays of buses and loss of time waiting at the station, knowing the location of the bus with the help of the application, they can save time or make decisions about their journey.

\section{Intelligent System Transportation}

Information technology allows elements within transport systems - cars, roads, traffic lights, traffic signs - to be intelligent by incorporating microchips and sensors to communicate with each other through wireless technologies. Intelligent applications bring significant improvements to the transport system, such as traffic reduction, safety gains and passenger comfort. Intelligent transport systems include a wide and growing range of technologies and applications, such as real-time traffic information systems, navigation systems, the collection of electronic traffic charges, finding parking places, etc.

The accelerated development of mobile technology, data acquisition and analysis, and their integration into different critical applications, such as transport systems, have the potential to create a more efficient, intelligent and secure infrastructure.

ITS deliver five key classes of benefits by: increasing safety; improving operational performance, particularly by reducing congestion; enhancing mobility and convenience; delivering environmental benefits; boosting productivity and expanding economic and employment growth [2].

\section{Challenges that can be addressed by the Intelligent Transport System}

Intelligent transport systems are represented by advanced applications of smart cities that aim to provide innovative services related to different modes of transport and traffic management to enable users to be better informed and to use the transport network in a secure, more coordinated and smarter way. There are many challenges that can be overcome by the intelligent transport system and some of them are presented below [3].

Traffic jam occurs as a result of the increase in the degree of agglomeration of a road. It is characterized by lower speeds, longer travel time and increased queues of cars. Traffic congestion occurs when too many cars require more space than available street capacity. It is caused by three main reasons: traffic incidents such as accidents, working roads, bad weather; traffic fluctuations due to a large number of cars on the road caused by special events or peak times; road conditions.

Searching for a free parking place in a metropolitan area is the daily concern of most drivers and is time consuming. It usually results in greater traffic congestion and air pollution by constantly searching for a parking place available in certain areas. In order to reduce such traffic congestion and improve driver comfort, several intelligent parking systems have developed to satisfy the parties involved [4].

\section{The benefits of Intelligent Transportation System}

Intelligent transport systems can provide important safety benefits. About 1,2 million people die on the road roads of the world every year. A wide range of its-based applications - from real-time traffic alerts to intersection-based collision avoidance to onboard systems, such as anti-lock braking, lane departure warning systems, collision avoidance and collision management systems - are intended primarily for safety. In fact, intelligent transport systems lead to a fundamental re-think of vehicle safety. Over the past 50 years, most of the developments in transport safety, such as the mandatory installation of safety belts in the years 1970 and the installation of airbags in the years 1980, have been designed to protect passengers in the event of an accident. 
The performance of a country's transport network can be improved by maximizing the capacity of existing infrastructure, without the need for additional construction of highways. Maximizing capacity is crucial because in almost all countries vehicle mileage increases dramatically exceed the increase in road capacity.

Intelligent Transport System improves driver mobility and comfort by reducing traffic congestion and maximizing operational efficiency of the transport system and providing real-time travel information and improved route selection and navigation. Probably the best known intelligent transport systems are applications such as satellite navigation of vehicles or other services providing real-time traffic information to drivers, either in vehicles or before they leave while planning to travel $[5,6]$.

\section{Web Technologies and Tools}

In this section are presented some web technologies and tools which were used for the application development.

Android Studio is the official Integrated Development Environment (IDE) for Android app development, based on IntelliJ IDEA . On top of IntelliJ's powerful code editor and developer tools, Android Studio offers even more features that enhance your productivity when building Android apps, such as: a flexible Gradle-based build system; a fast and feature-rich emulator; a unified environment where you can develop for all Android devices; apply changes to push code and resource changes to your running app without restarting your app; extensive testing tools and frameworks; lint tools to catch performance, usability, version compatibility, and other problems.

The Firebase Realtime Database is a cloud-hosted database. Data is stored as JSON and synchronized in realtime to every connected client. When you build cross-platform apps with our iOS, Android, and JavaScript SDKs, all of your clients share one Realtime Database instance and automatically receive updates with the newest data. Store and sync data with our NoSQL cloud database. Data is synced across all clients in realtime, and remains available when your app goes offline.

\section{Application Architecture and Implementation}

The project is structured in three main components: The Android application designed for the bus driver, the Android application designed for users and the Firebase database. Each component has a well-defined role to be detailed below. They communicate with each other ensuring that the application works properly.

The main functionality of the driver component is to provide its location in real time. The data is inserted into the database and from there the user application extracts it to provide this information to users.

The designated passenger application has several functionalities, among which the most important is to display the location of the bus in real time. The user can also see its location on the map. Another functionality is to see the entire bus route on the map with marked stations. In the idea of smart mobility, the search function for a route has also been added, so that the application would be useful for user, even if he does not use a public transport mode, but a car.

Firebase is a platform for developing web and mobile applications that provides developers with a wide range of services and tools to develop high-quality applications and to increase the user base.

The first developed application, the bus driver application, has the most important functionality, and that is to store its location in the database in real time, with the data constantly updated. Most applications use user authentication methods to save their preferences and to be possible that the administrator monitor them. The authentication method used in this application is based on email and password.

The primary activity of the application, MapsActivity, is triggered when a user logs or logins. This is intended to display the actual bus location on the map and to store this data, latitude and longitude in the database so that these data can then be taken over by the traveller's application.

The data is stored in Firebase database. In the users key, there are two other keys, BusDrivers and Travelers, containing user id. In this way, each user has a different id within which the coordinates of his location are located. Key 0 is latitude and key 1 logon.

$40 \quad$ Journal of E-Technology Volume 10 Number 2 May 2019 




Figure 1. The use case diagram of application

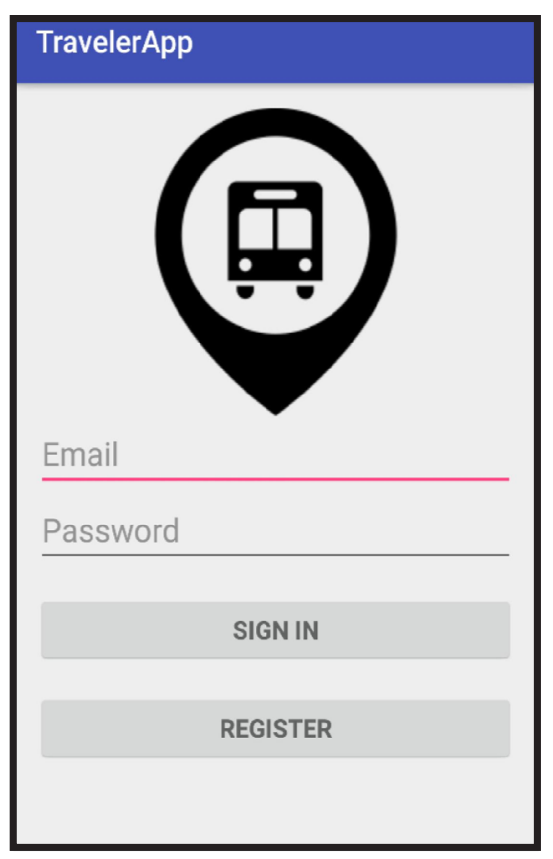

Figure 2. The interface of the registration and logging activity

The second developed application is that of the traveller and its role is to provide users with real-time information about the desired bus position. The need for this application comes from the desire of citizens to have an intelligent transport system. The structure of the application is similar to that of bus drivers. Also, when the program is run, the recording/logging activity will be 




Figure 3. The database structure

displayed. The way this work is implemented is the same as in the drivers' application. Of course, the data will be stored inside the passenger key. After the user has registered or logged in, it is forwarded to the main activity of the application, MapsActivity. In the first instance, the map will be displayed with the current traveler location. The traveler's location is implemented in a similar way as in the first application. In addition to displaying the map in the main activity, a menu with a many options is designed. The menu is implemented with a DrawerLayout. The menu interface can be seen in the following illustration.

The most important application functionality is implemented in the getBusLocation function(). The location of the bus is taken from the database. A reference to the database is created, and it points to where the bus application stores its data in the form of latitude and longitude. To this reference, an Eventlistener is added which implements the onDataChanged (DataSnapshot) public function. Check if data exists and if the result is positive, create an object list map that receives the data value using the getValue method(). Two variables, locationLat and locationLong, are initialized with zero, and are used as follows. If the first item of the lists is not null, the variable locationLat is assigned the first value in the list. The same happens for the second item of the list. If he is not null, the value of the variable is assigned to the locationLong variable. Assignments are carried out in this way, as latitude lies in the database on key 0 and longitude on key 1 . The values extracted are assigned to a new variable of the type LatLng.

The data is downloaded using the DownloadRawData() method that extends the AsyncTask that allows the correct and easy use of interface wires. For the asynchronous task to be executed two methods are implemented: DoInBackground() and onPostexecute(). Data is read line-by-line with a bufferReader and inserted into a string buffer.

Once the data is received, they will be divided by the parseJson(data) function. Class Route is defining seven important variables which are helping in drawing the route. These are: distance and duration, which have two parameters, text and value, two string variablesstartAddress and endAddress; two variables startLocation and endLocation which have the latitude and longitude; and a points list. Following is an example of a JSON file.

\begin{tabular}{llllll}
\hline 42 & Journal of E-Technology Volume & 10 & Number 2 & May 2019 \\
\hline
\end{tabular}




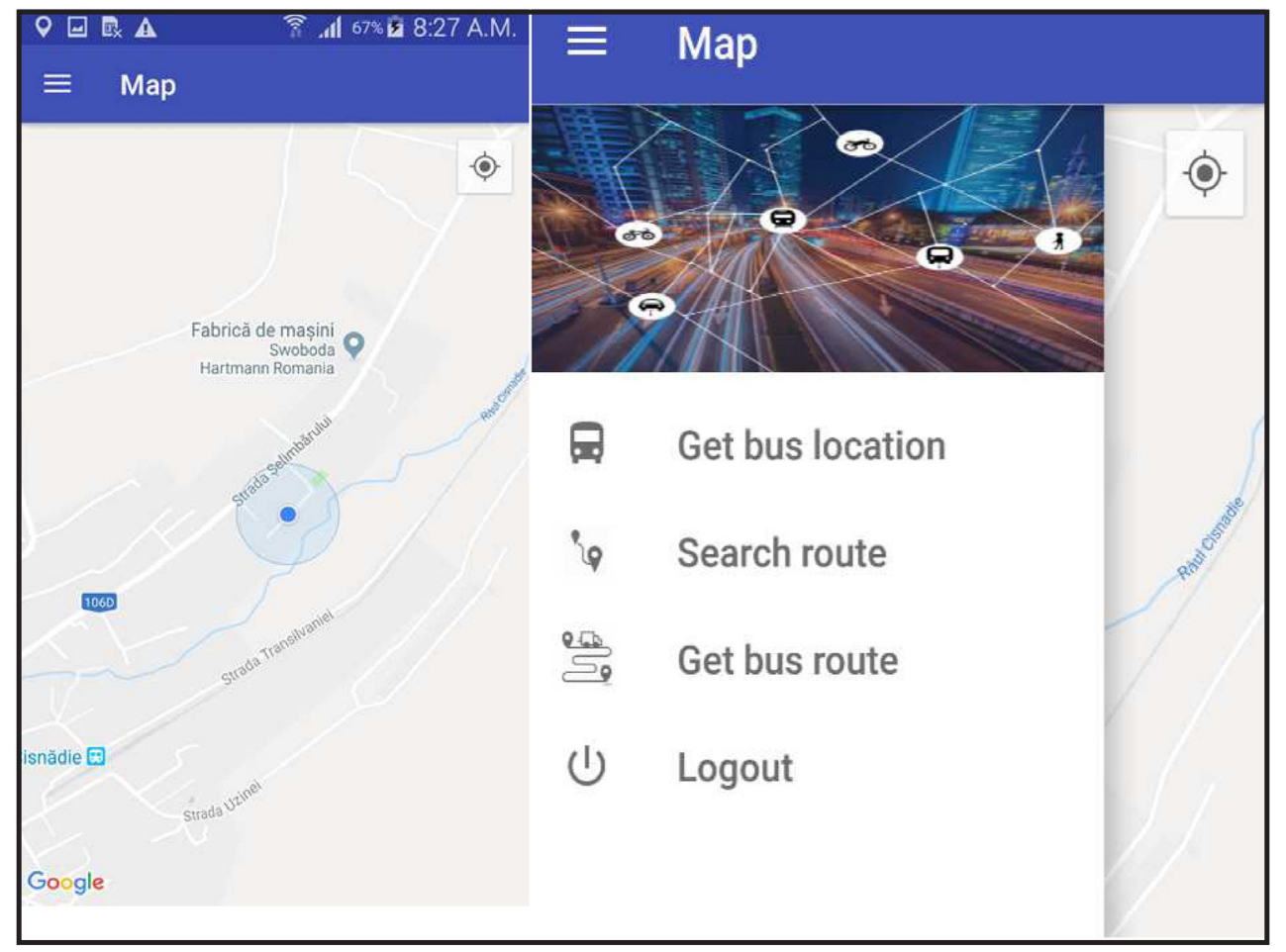

Figure 4. Display the map with the user's location (left); The application menu (right)

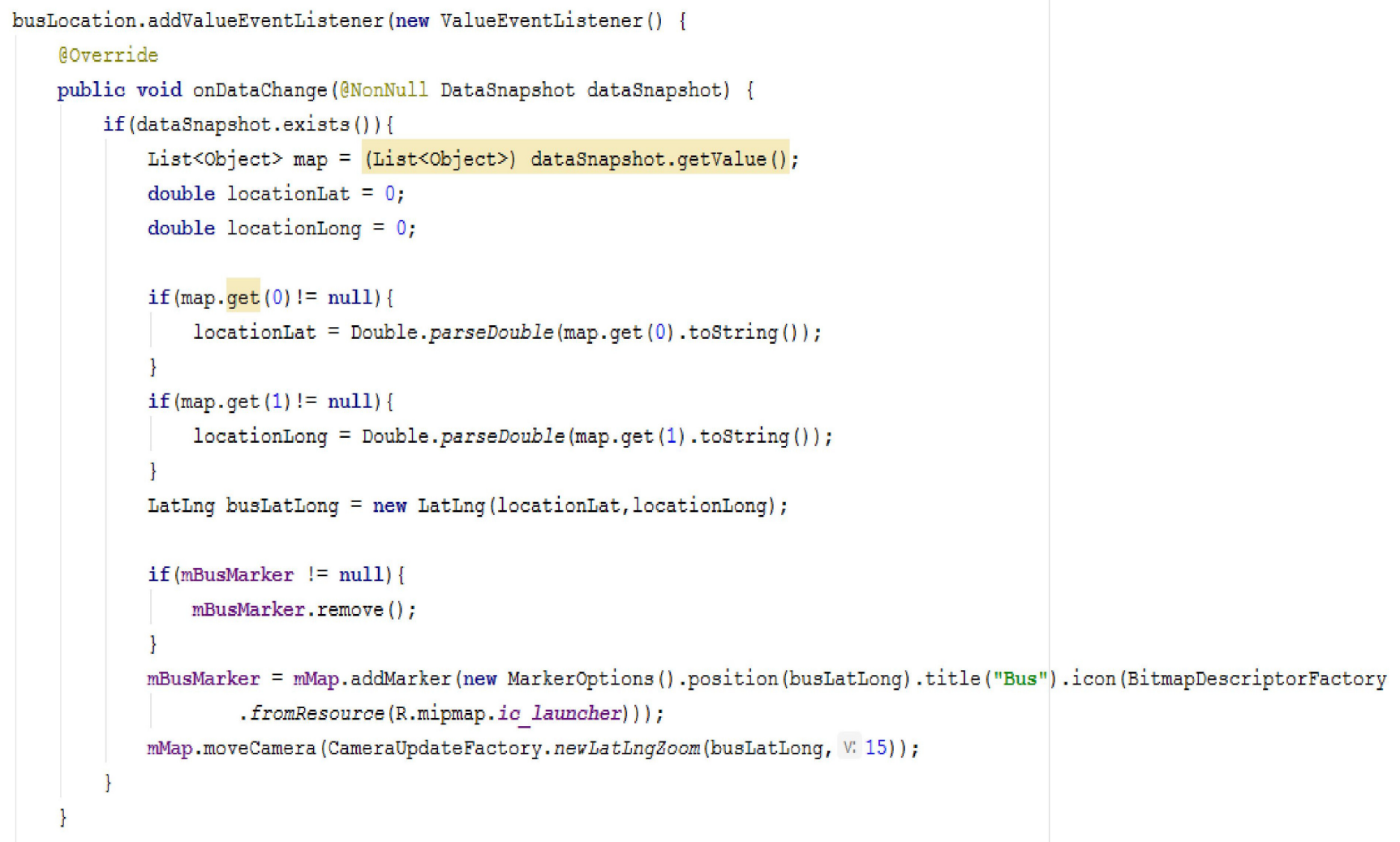




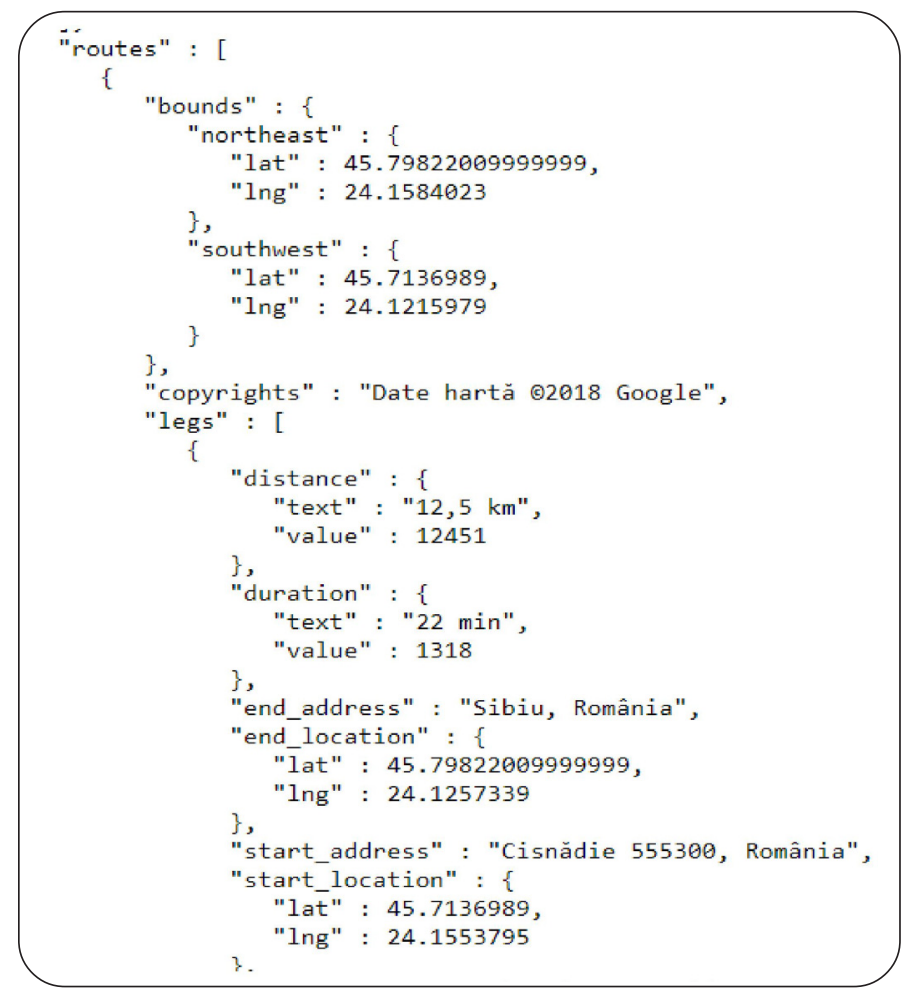

Figure 5. Example of JSON file

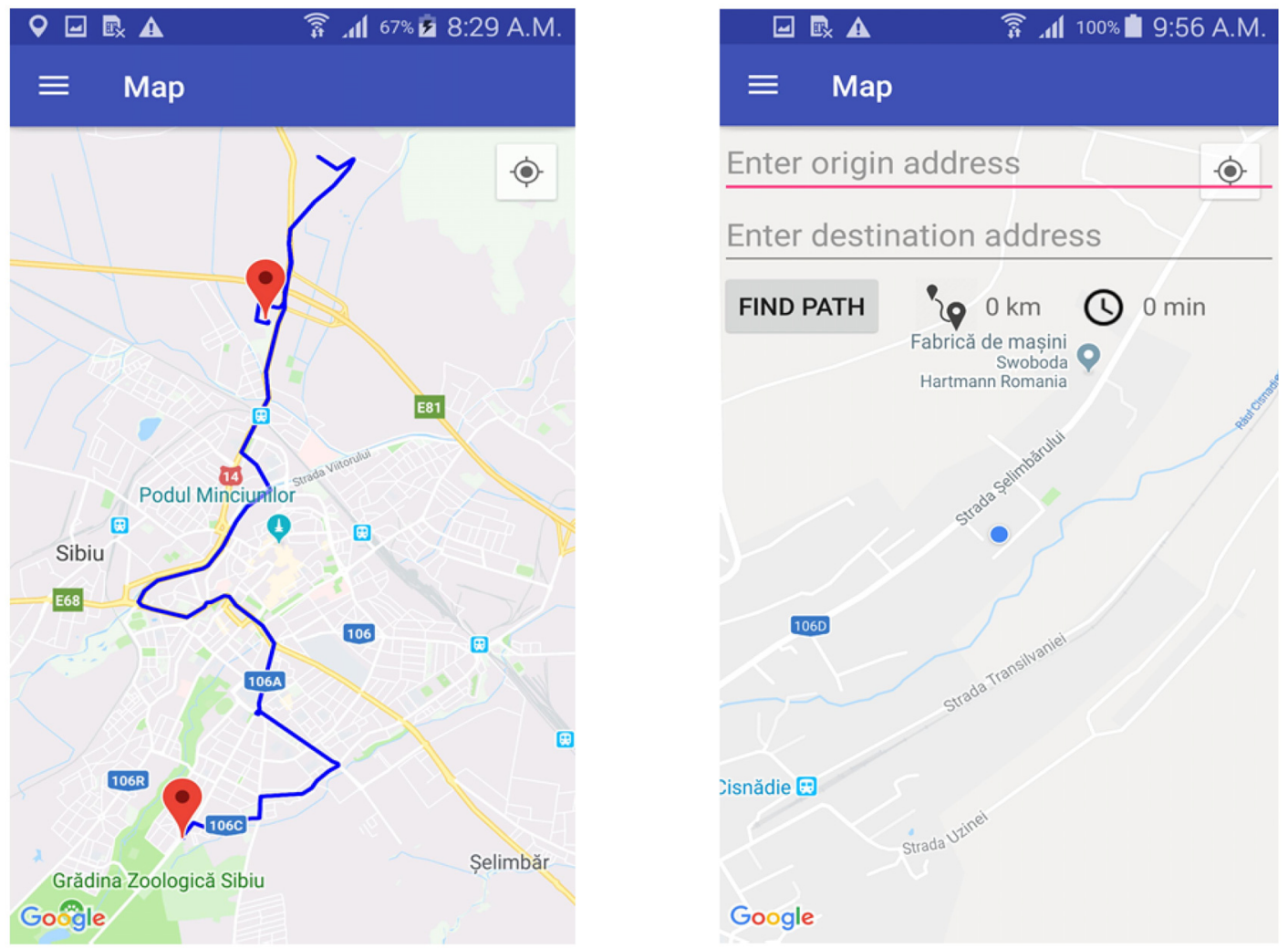

Figure 6. Finding and displaying a route 


\section{Conclusions and further Development}

Intelligent transport systems play a very important role in the lives of the inhabitants of major cities. It can improve the way people travel and even the quality of life through the many benefits. Several benefits of this system are reducing the traffic, reducing gas emissions, increasing safety, improving comfort and mobility and many others. The objective of the project was to create an application to help the city's inhabitants use public transport. This would be a solution to reducing the number of vehicles present daily in traffic.

Of course somebody could critic that there are some implementation solutions in high developed countries. But, our solution is appropriate especially in countries less developed, where municipalities did not invest yet in such solutions. Further developments are also important to improve user experience. The application developed at the current stage takes data from a single bus and transmits it to users. It is also intended to develop this application by the existence of several buses in the database.

Another development is the display of the remaining time until the bus reaches the station desired by the user and even the connection of station connections.

Finally, the possibility to choose the language of choice, as tourists are the ones most in need of such help, not knowing the routes or locations of the city.

\section{References}

[1] Van Audenhove, F. J., Korniichuk, O., Dauby, L., Pourbaix, J. (2014). The future of urban mobility 2.0: Imperatives to shape extended mobility ecosystems of tomorrow.

[2] Tomaszewska, E. J., Florea, A. (2018). Urban smart mobility in the scientific literature-bibliometric analysis. Engineering Management in Production and Services, 10 (2) 41-56.

[3] Guerrero-Ibáñez, J., Zeadally, S., Contreras-Castillo, J. (2018). Sensor technologies for intelligent transportation systems. Sensors, 18 (4) 1212.

[4] Marcu, S. D., Florea, A. (2018, September). Smart parking system - Another way of sharing economy provided by private institutions. In: Proceedings of the Thirteenth International Conference on Digital Information Management (ICDIM), Berlin, Germany (24-26).

[5] EU Road Safety Policy Framework 2021-2030 - Next steps towards “Vision Zero”, Brussels, 19.6.2019, https://ec.europa.eu/ transport/road_safety/home_en

[6] Yatskiv, I., Savrasovs, M., Udre, D., Ruggeri, R. (2017). Review of intelligent transport solutions in Latvia. Transportation Research Procedia, 24, 33-40. 